Global Journal of Pure and Applied Mathematics.

ISSN 0973-1768 Volume 11, Number 5 (2015), pp. 3743-3749

(C) Research India Publications

https://dx.doi.org/10.37622/GJPAM/11.5.2015.3743-3749

\title{
Algorithmic Approach for Solving Transportation Problems
}

\author{
A. Akilbasha*1, G. Natarajan², P. Pandian ${ }^{3}$ \\ School of Advanced Sciences, VIT University, Vellore-14.
}

\begin{abstract}
A new method called modified zero suffix method is proposed for finding an optimal solution for transportation problems in single stage. The solution procedure is given with numerical examples. As this method is very easy to understand and apply, it will help the managers in logistics related issues by aiding them in the decision making process and providing an optimal solution in a simple and effective manner.
\end{abstract}

Keywords: Direct method, sources, destinations, optimal solution, transportation problem.

\section{Introduction}

The transportation problem is a special class of linear programming problem which has applications in industry, communication network, planning, scheduling and transportation etc. The transportation problem deals with shipping commodities from different sources to various destinations. The objective of the transportation problem is to determine the shipping schedule that minimizes the total shipping cost while satisfying supply and demand limits. The algorithm of the approach is detailed with suitable numerical examples. Further comparative studies of the new technique with other existing algorithms are established by means of sample problems.

\section{Literature Review}

In real world applications, the supply and demand quantities in the transportation problem are sometimes hardly specified precisely because of changing economic conditions. It was first studied by F. L. Hitchcock in 1941, then separately by Koopmans [10], and finally placed in the framework of linear programming and solved by simplex method by Dantzig [4]. Chandra and Saxena [3] proposed time minimizing 
transportation problem with impurities. Abdul Quddoos et al. [1] proposed a new method named ASM-Method is proposed for finding an optimal solution for a wide range of transportation problems, directly. Pandian and Natarajan [12,13] studied a new method namely, blocking method for finding an optimal solution to BTPs and also they proposed another method namely, blocking zero point method for finding all efficient solutions to a bottleneck-cost transportation problem. Shimshak et al. [15] proposed a modification of vogel's approximation method through the use of heuristics. Recently, Akilbasha et al. [2] have proposed a new method for finding an optimal solution for fully fuzzy BCTPs. And also so many Researchers like Gaurav Sharma et al. [5] solved the Transportation Problem with the help of Integer Programming Problem. Gupta [6] proposed a new method for solving Time-Cost Transportation Problem. Issermann [8] proposed a method for solving linear bottleneck transportation problem. Kirca and Satir [9] find a heuristic for obtaining an initial solution for the transportation problem. Nagoor Gani and Abdul Razak [11] solved the fuzzy transportation problems in two stages. Hasan [7] studied direct method for finding optimal solutions of transportation problems are not always reliable. Prakash [14] studied on minimizing the duration of the transportation. Several sorts of methods have been established for finding an optimal solution to the transportation problems.

\section{Proposed Method}

In this paper, a new method called modified zero suffix method is proposed for finding an optimal solution for transportation problems with equality constraints in a single stage. This method is very easy to understand and apply. This method has much easier heuristic approach for finding an optimal solution directly with lesser number of iterations and very easy computations. Three numerical examples are provided to prove my claim.

This paper is organized as follows: In section 4, general format of transportation problem, some basic definitions are listed. In section 5, our proposed method for finding an optimal solution to transportation problem by using modified zero suffix method is presented. In section 6, numerical examples are solved. The conclusion is discussed in section 7 .

\section{Transportation problem with equality constraints}

Consider the following transportation problem with equality constraints.

Minimize $Z=\sum_{\mathrm{i}=1}^{m} \sum_{\mathrm{j}=1}^{n} c_{\mathrm{ij}} x_{\mathrm{ij}}$

Subject to

$\sum_{j=1}^{n} x_{i j}=a_{i}, \quad i=1,2, \ldots ., m$

$\sum_{i=1}^{m} x_{i j}=b_{j}, j=1,2, \ldots ., n$

$x_{\mathrm{ij}} \geq 0$, for all $i$ and $j$ are integers, 
Where $m$ is the number of supply points; $n$ is the number of demand points; $x_{\mathrm{ij}}$ is the number of units shipped from supply point $\mathrm{i}$ to demand point $\mathrm{j}$;

$c_{\mathrm{ij}}$ is the cost of shipping one unit from supply point $\mathrm{i}$ to the demand point $\mathrm{j}$;

$a_{i}$ is the supply at supply point $\mathrm{i}$ and $b_{j}$ is the demand at demand point $\mathrm{j}$.

Now, the above problem can be put in the following transportation table.

\begin{tabular}{|c|c|c|c|c|c|}
\hline & \multirow{3}{*}{$\begin{array}{l}\text { Supply } \\
a_{1} \\
a_{2}\end{array}$} \\
\hline & $c_{11}$ & $c_{12}$ & $\ldots$ & $c_{1 \mathrm{n}}$ & \\
\hline & $c_{21}$ & $c_{22}$ & $\ldots$ & $c_{2 \mathrm{n}}$ & \\
\hline & $\equiv$ & $\equiv$ & $\equiv$ & $\equiv$ & $\equiv$ \\
\hline & $c_{\mathrm{m} 1}$ & $c_{\mathrm{m} 2}$ & . & $c_{\mathrm{m} \mathrm{n}}$ & $a_{m}$ \\
\hline mand & $b_{1}$ & $b_{2}$ & . & $b_{\mathrm{n}}$ & \\
\hline
\end{tabular}

\section{1 feasible Solution}

Any set of non-negative allocations of a transportation problem which satisfies the row sum or column sum (if the problem is unbalanced ) or both (if the problem is balanced) is called a feasible solution.

\subsection{Optimal Solution}

A feasible solution which minimizes the total shipping cost is said to be optimal solution.

\subsection{Balanced and Unbalanced Transportation Problem}

A Transportation Problem (TP) is said to be balanced if the total supply from all sources equals to the total demand in the destinations (i.e.) $\sum_{i=1}^{m} a_{i}=\sum_{j=1}^{n} b_{j}$, Otherwise it is unbalanced symbolically $\sum_{i=1}^{m} a_{i} \neq \sum_{j=1}^{n} b_{j}$.

\section{Modified Zero Suffix Method}

We, now introduce a new method called the modified zero suffix method for finding an optimal solution to a transportation problem.

The method proceeds as follows.

Step 1: Construct the transportation table for the given TP.

Step 2: Subtract each row entries of the transportation table from the row minimum and then subtract each column entries of the resulting transportation table after using the Step 1 from the column minimum. 
Step 3: Check if each column demand is less than to the sum of the supplies whose reduced costs in that column are zero. Also, check if each row supply is less than to sum of the column demands whose reduced costs in that row are zero. If so, go to Step 7. (Such reduced table is called the allotment table). If not, go to Step 4.

Step 4: Draw the minimum number of horizontal lines and vertical lines to cover all the zeros of the reduced transportation table such that some entries of row(s) or / and column(s) which do not satisfy the condition of the Step3 are not covered.

Step 5: Develop the new, revised and reduced transportation table as follows:

(i) Find the smallest entry of the reduced cost matrix not covered by any lines.

(ii) Subtract this entry from all the uncovered entries and add the same to all entries lying at the intersection of any two lines and then, go to Step 3.

Step 6: Repeat the steps 3 to 5 till the step 3 is satisfied.

Step 7: In the reduced cost matrix there will be at least one zero in each row and column, then find the suffix value of all the zeros in the reduced cost matrix by following simplification, the suffix value is denoted by $S$, Therefore $S=\{$ Add all the costs (which is greater than zero) of adjacent rows and columns corresponding to each zeros / No. of costs added $\}$

Do allocation according to the following rules:

a) Choose the maximum of $\mathrm{S}$, if it has one maximum value then first supply to that demand corresponding to the cell. If it has more equal values then select $\left\{a_{i}, b_{j}\right\}$ and supply to that demand maximum possible.

b) Then allot the next maximum value of $\mathrm{S}$ and continuing the process till all the supply and demands are fully supplied and fully received.

\section{Numerical examples}

Example 6.1 Consider the following cost minimizing transportation problem with four sources and three destinations

\begin{tabular}{|l|c|c|c|c|c|}
\hline & S1 & S2 & S3 & S4 & Supply \\
\hline D1 & 13 & 18 & 30 & 8 & 8 \\
\hline D2 & 55 & 20 & 25 & 40 & 10 \\
\hline D3 & 30 & 6 & 50 & 10 & 11 \\
\hline Demand & 4 & 7 & 6 & 12 & \\
\hline
\end{tabular}

By applying the proposed Modified Zero Suffix Method allocations are obtained as follows: 


\begin{tabular}{|l|c|c|c|c|c|}
\hline & S1 & S2 & S3 & S4 & Supply \\
\hline D1 & 13 & 18 & 30 & 8 & 8 \\
& $\mathbf{( 4 )}$ & $\mathbf{( 3 )}$ & & $(\mathbf{1})$ & \\
\hline D2 & 55 & $\begin{array}{c}20 \\
(\mathbf{4})\end{array}$ & $\begin{array}{c}25 \\
(\mathbf{6})\end{array}$ & 40 & 10 \\
& & 6 & 50 & 10 & 11 \\
\hline D3 & 30 & & & $\mathbf{( 1 1 )}$ & \\
\hline Demand & 4 & 7 & 6 & 12 & \\
\hline
\end{tabular}

The minimum transportation cost associated with this solution is

$$
\begin{aligned}
Z & =\$(13 \times 4+18 \times 3+8 \times 1+20 \times 4+25 \times 6+10 \times 11) \\
& =\$(52+54+8+80+150+110) \\
& =\$ 454
\end{aligned}
$$

Commits: The existing zero suffix method gives the optimal solution is $\$ 476$ but the proposed modified zero suffix method shows that the optimal solution is $\mathbf{\$ 4 5 4}$.

Example 6.2 Consider the following cost minimizing transportation problem with six sources and four destinations:

\begin{tabular}{|c|c|c|c|c|c|c|c|}
\hline & S1 & $\mathrm{S} 2$ & S3 & $\mathrm{S} 4$ & S5 & S6 & Supply \\
\hline D1 & 9 & 12 & $\begin{array}{c}9 \\
\text { (5) }\end{array}$ & 6 & 9 & 10 & 5 \\
\hline D2 & 7 & $\begin{array}{c}3 \\
\text { (4) }\end{array}$ & 7 & 7 & 5 & $\begin{array}{c}5 \\
(2)\end{array}$ & 6 \\
\hline D3 & 6 & 5 & $\begin{array}{c}9 \\
\text { (1) }\end{array}$ & 11 & $\begin{array}{c}3 \\
(\mathbf{1})\end{array}$ & 11 & 2 \\
\hline D4 & $\begin{array}{c}6 \\
\text { (4) }\end{array}$ & 8 & 11 & $\begin{array}{c}2 \\
(2)\end{array}$ & $\begin{array}{c}2 \\
(3)\end{array}$ & 10 & 9 \\
\hline Demand & 4 & 4 & 6 & 2 & 4 & 2 & \\
\hline
\end{tabular}

\begin{tabular}{|l|c|c|c|c|c|c|c|}
\hline & S1 & S2 & S3 & S4 & S5 & S6 & Supply \\
\hline D1 & 9 & 12 & 9 & 6 & 9 & 10 & 5 \\
\hline D2 & 7 & 3 & 7 & 7 & 5 & 5 & 6 \\
\hline D3 & 6 & 5 & 9 & 11 & 3 & 11 & 2 \\
\hline D4 & 6 & 8 & 11 & 2 & 2 & 10 & 9 \\
\hline Demand & 4 & 4 & 6 & 2 & 4 & 2 & \\
\hline
\end{tabular}

By applying Modified Zero Suffix Method allocations are obtained as follows:

The minimum transportation cost associated with this solution is 


$$
\begin{aligned}
\mathrm{Z} & =\$(9 \times 5+3 \times 4+5 \times 2+9 \times 1+3 \times 1+6 \times 4+2 \times 2+2 \times 3) \\
& =\$(45+12+10+9+3+24+4+6) \\
& =\$ 113
\end{aligned}
$$

Commits: The modified zero suffix method shows that the optimal solution is $\mathbf{\$ 1 1 3}$ but the existing zero suffix method gives the optimal solution is \$136.

Example 6.3 Consider the following cost minimizing transportation problem with five sources and four destinations:

\begin{tabular}{|l|l|l|l|l|l|l|}
\hline & S1 & S2 & S3 & S4 & S5 & Supply \\
\hline D1 & 4 & 3 & 1 & 2 & 6 & 80 \\
\hline D2 & 5 & 2 & 3 & 4 & 5 & 60 \\
\hline D3 & 3 & 5 & 6 & 3 & 2 & 40 \\
\hline D4 & 2 & 4 & 4 & 5 & 3 & 20 \\
\hline Demand & 60 & 60 & 30 & 40 & 10 & \\
\hline
\end{tabular}

By applying Modified Zero Suffix Method allocations are obtained as follows:

\begin{tabular}{|l|l|l|l|l|l|l|}
\hline & S1 & S2 & S3 & S4 & S5 & Supply \\
\hline D1 & $\begin{array}{l}4 \\
(\mathbf{1 0})\end{array}$ & 3 & $\begin{array}{l}1 \\
\mathbf{( 3 0 )}\end{array}$ & $\begin{array}{l}2 \\
\mathbf{( 4 0 )}\end{array}$ & 6 & 80 \\
\hline D2 & 5 & $\begin{array}{l}2 \\
\mathbf{6 0})\end{array}$ & 3 & 4 & 5 & 60 \\
\hline D3 & $\begin{array}{l}\mathbf{( 3 0 )} \\
\text { D }\end{array}$ & 5 & 6 & 3 & $\begin{array}{l}2 \\
\mathbf{( 1 0 )}\end{array}$ & 40 \\
\hline D4 & $\begin{array}{l}2 \\
\mathbf{( 2 0 )}\end{array}$ & 4 & 4 & 5 & 3 & 20 \\
\hline Demand & 60 & 60 & 30 & 40 & 10 & \\
\hline
\end{tabular}

The minimum transportation cost associated with this solution is

$$
\begin{aligned}
Z & =\$(4 \times 10+1 \times 30+2 \times 40+2 \times 60+3 \times 30+2 \times 10+2 \times 20) \\
& =\$(40+30+80+120+90+20+40) \\
& =\$ 420
\end{aligned}
$$

Commits: The modified zero suffix method and the existing zero suffix method both gives the optimal solution is $\mathbf{\$ 4 2 0}$.

\section{Conclusion}

The optimal solution obtained by the Modified Zero Suffix Method gives the best optimal solution to the transportation problems by comparing to the existing Zero 
Suffix Method. This proposed Modified Zero Suffix Method helps the decision makers to select the best optimal solution.

\section{References}

[1] Abdul Quddoos, Shakeel Javaid, Khalid, M. M., 2012, "A New Method for Finding an Optimal Solution for Transportation Problems," International Journal on Computer Science and Engineering (IJCSE), Vol. 4(7), pp. 12711274.

[2] Akilbasha, A., Natarajan, G., and Pandian, P., 2014, "A New Method for Solving Fully Fuzzy Bottleneck-Cost Transportation Problems," The International Journal Of Science \& Technoledge, Vol. 2, pp. 63-71.

[3] Chandra, S., and Saxena, P.K., 1987, "Time Minimizing Transportation Problem with impurities," Asia- Pacific J. Op. Res., Vol. 4, pp. 19- 27.

[4] Dantzig, G.B., 1963, Linear Programming and Extensions, Princeton University Press, Princeton, N J.

[5] Gaurav Sharma, Abbas, S. H., Vijay kumar Gupta, June 2012, "Solving Transportation Problem with the help of Integer Programming Problem," IOSR Journal of Engineering, Vol. 2(6) pp. 1274-1277.

[6] Gupta, R., 1977, “Time-Cost Transportation Problem," Econ-math overview, Vol. 4, pp. 431-443.

[7] Hasan, M.K., 2012, "Direct Methods for Finding Optimal Solution of a Transportation Problem are not Always Reliable," International Refereed Journal of Engineering and Science, Vol. 1(2), pp. 46-52.

[8] Issermann, H., 1984, "Linear bottleneck transportation problem," Asia Pacific Journal of Operational Research, Vol. 1, pp. 38 - 52.

[9] Kirca, O., and Satir, A., 1990, "A heuristic for obtaining an initial solution for the transportation problem," Journal of Operational Research Society, Vol. 41(9), pp. 865-871.

[10] Koopmans, T.C., 1949, “Optimum Utiliztion of Transportation System,” Eco. Supplement, Vol 17.

[11] Nagoor Gani, A., and Abdul Razak, K., 2006, "Two stage fuzzy transportation problem,” Journal of Physical Sciences, Vol. 10, pp. 63-69.

[12] Pandian, P., and Natarajan, G., 2011, "A New Method for Solving BottleneckCost Transportation Problems," Int. Math. Forum, Vol. 6(10), pp. $451-460$.

[13] Pandian, P., and Natarajan, G., 2010, "A new method for finding an optimal solution for transportation problems," Int. J. of Math.Sci. \&Engg.Appls., Vol. 4, pp. 59-65.

[14] Prakash, S., 1982, "On minimizing the duration of transportation," Proceedings of the Indian Academy of Sciences-Mathematical Sciences, Vol. 91, pp. 53-57.

[15] Shimshak, D.G., Kaslik, J.A., and Barclay, T.D., 1981, "A modification of Vogel's approximation method through the use of heuristics," INEOR, Vol. 19, pp. 259-263. 
